\title{
Deep Graph Convolutional Networks for Wind Speed Prediction
}

\author{
Tomasz Stańczyk and Siamak Mehrkanoon \\ Maastricht University - Department of Data Science and Knowledge Engineering \\ Paul-Henri Spaaklaan 1, 6229 EN Maastricht - The Netherlands
}

\begin{abstract}
In this paper, we introduce a new model for wind speed prediction based on spatio-temporal graph convolutional networks. Here, weather stations are treated as nodes of a graph with a learnable adjacency matrix, which determines the strength of relations between the stations based on the historical weather data. The self-loop connection is added to the learnt adjacency matrix and its strength is controlled by additional learnable parameter. Experiments performed on real datasets collected from weather stations located in Denmark and the Netherlands show that our proposed model outperforms previously developed baseline models on the referenced datasets.
\end{abstract}

\section{Introduction and Related Work}

Deep learning based models have been successfully applied for weather elements forecasting [1]3]. Although Long Short-Term Memory (LSTM) recurrent networks [4], work well for time series prediction [5], they do not explicitly include spatial relations within the data. Authors in [6] combined LSTM with convolutions to create ConvLSTM, which was used for precipitation tasks, while also capturing the data spatial structure. The authors in 3 introduced convolutional neural network $(\mathrm{CNN})$ models based on 1D-CNNs for multi-source 1D weather data, 2D- and 3D-CNNs to process the tensor-form 3D weather data. In this way, the spatial-temporal relations were extracted with 2D and 3D CNNs. The authors in $[7]$ proposed using depthwise-separable convolutions to process different dimensions of the input tensor and then concatenating resulting tensor along single dimension. However, all the CNN-based models above discard the spatial relations between the cities (weather stations). In other words, these approaches process input tensor, where neighborhood of the cities is determined only by the order of the cities in the tensor or the dataset.

Graph convolution networks (GCNs), which are a particular type of graph neural networks 8 can generalize CNNs to work on graphs rather than on regular grids [9]. In particular, it enables incorporating the neighbor relation information, e.g. through an adjacency matrix of a graph. The authors in 10 introduced weighted graph convolutional LSTM architecture, which combines LSTM with matrix multiplications replaced with graph convolutions with a single (one for the whole model), learnable adjacency matrix. In [11], the authors created a graph based on the wind farms and for each node of the graph, temporal features were extracted with LSTMs. 
In this work, we treat weather stations and their corresponding weather variable values from different time steps as a spatio-temporal graph, as presented in Fig. 11(c). Here, we develop our own novel model from ST-GCN 12 and 2s-AGCN [13 architectures which were successful on skeleton-based action recognition tasks. In particular, we include learnable parameter controlling the self-connection strength of the learnt adjacency matrix and normalize the matrix.

This paper is organized as follows. Section 2 presents the proposed model and relevant details. Section 3 describes the conducted experiments with corresponding results. Finally, conclusions are drawn in Section 4

\section{Methods}

In this work we experiment on two Dutch and Danish datasets. The data consists of historical observations (time steps) for several cities and several weather variables. The aim is to predict the wind speed values for selected cities for several time steps ahead. At a single time step, we treat the input cities as a graph where each city is a node in the graph. Node attributes are then weather variables for the first layer of the network and features encoded by the network in the next layers. For the cities shown in Fig. 11(a), at a single time step, the corresponding spatial graph could be perceived as in Fig. 1(b).

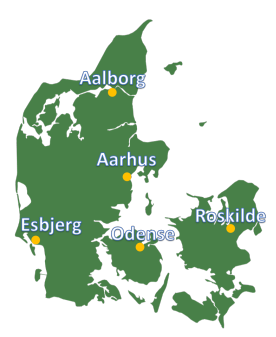

(a)

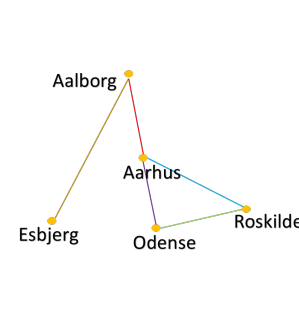

(b)

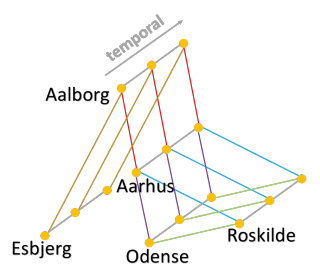

(c)

Fig. 1: (a) Map with the Danish weather stations. Possible: (b) spatial graph; (c) spatial-temporal graph; built from Danish weather stations.

In practice, time series of historical data involve multiple time steps. Therefore, we include this information by expanding the previous graph into a spatialtemporal graph as shown in Fig. 1.(c). Two types of convolutions are included in our proposed models, i.e. graph spatial convolution and temporal convolution.

\subsection{Graph Spatial Convolution}

Spatial convolution aggregates the information from the spatial neighbors of the graph. The graph is represented by an adjacency matrix. Following the lines of 10 13, we make the adjacency matrix learnable. During the optimization, the network learns the graph spatial connections between the weather stations. It should be noted that since all the entries are learnt in an end-to-end fashion, the adjacency matrix is not symmetric. The learnable adjacency matrix is further 
transformed during the training. Operations similar to those from GCNs of [14], involving adding the self-loop connection and normalization with degree matrix are applied. To the best of our knowledge, it is the first time to apply such transformations over the adjacency matrix which is learnable. The new, transformed matrix is created as follows. Self-loop connection in the form of an identity matrix is added to the learnable adjacency matrix: $\hat{A}=A+\gamma I$. Here, $\gamma$ is a learnable scalar parameter which lets the network decide about the strength of the imposed self-loop connection. The resulting matrix $\hat{A}$ is then scaled as follows: $\hat{A}=\frac{\hat{A}-\hat{A}_{\min }}{\hat{A}_{\max }-\hat{A}_{\text {min }}}$. A diagonal node degree matrix $\hat{\mathrm{D}}$ is computed based on the normalized matrix: $\hat{D}_{i i}=\sum_{j} \hat{A}_{i j}$. Next, we apply the symmetric normalization 14]: $\hat{D}^{-\frac{1}{2}} \hat{A} \hat{D}^{-\frac{1}{2}}$. The resulting transformed matrix is used for the graph convolution operation. Input data tensor $\mathcal{X}_{i n}$ with the shape of $C \times T \times V$, where $C=$ \#channels (weather variables), $T=$ \#timesteps and $V=\#$ graph vertices (cities) is reshaped into a matrix $X_{i n}$ with dimension of $C T \times V$. The graph convolution is initially performed by multiplying the reshaped input matrix with the transformed adjacency matrix:

$$
X_{\text {out }}=X_{\text {in }}\left(\hat{D}^{-\frac{1}{2}} \hat{A} \hat{D}^{-\frac{1}{2}}\right) .
$$

Next, the output matrix $X_{\text {out }}$ is reshaped back into a tensor $\mathcal{X}_{\text {out }}$ with the shape of $C \times T \times V$. Finally, $1 \times 1$ convolution is performed in order to combine the features channel-wise and to increase the number of channels.

\subsection{Temporal Convolution}

Temporal convolution aggregates the information from the temporal neighbors in the graph. For each separate node and its features, information from the next and/or previous time step is included. The temporal convolution is implemented as a regular 2D convolution with filter of size $k \times 1$. The value of $k$ is set to 3 , as this value was experimentally found to be optimal value for our models regarding their performance on the validation sets of the used datasets.

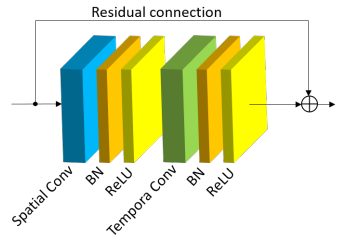

(a)

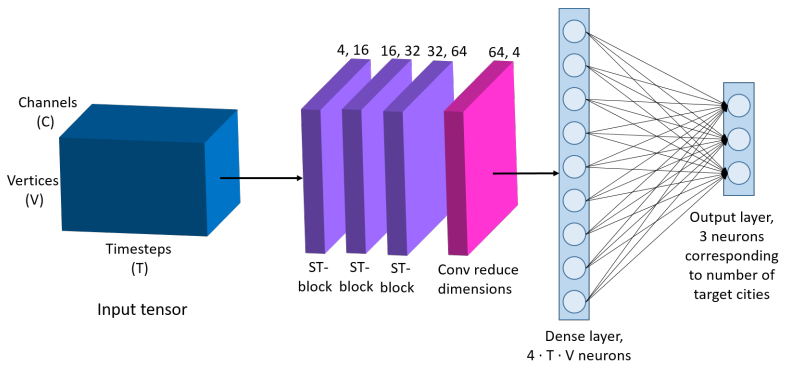

(b)

Fig. 2: (a) Spatio-temporal block (ST-block) used in our models. Figure inspired by [13. (b) Overview of the proposed model(s). The numbers above the blocks indicate the number of input and output channels respectively.

Using spatial convolution and temporal convolution operations, we create a spatio-temporal block (ST-block). As presented in Fig. 2(a), both spatial 
and temporal convolution are followed by a batch normalization (BN) layer and a ReLU activation function. In addition, a residual connection is added over the whole block. Each block contains its own, separate, learnable adjacency matrix. We stack three spatio-temporal blocks with the following number of output channels: 16,32 and 64 . The last block is followed by $1 \times 1$ convolution which reduces the number of channels to 4 before flattening. A fully connected (FC) layer is added at the end of the network to obtain wind speed predictions for target cities. The model architecture is presented in Fig. 2(b). We call our proposed model WeatherGCNet. The following setup is applied in the training phase. Batch size is set to 64 . Adam optimizer is used with the default value of learning rate set to 0.001 . The number of input timesteps $T$ processed by our models is tuned on validation sets and set to 30 .

\section{Experimental Results}

We consider two datasets for the task of wind speed prediction. The data comes from weather stations located in Denmark and the Netherlands. The datasets contain hourly measurements of several weather variables for several Danish and Dutch cities. In this study, the prediction targets are set to wind speed of Esbjerg, Odense, Roskilde for the Danish dataset and Schiphol, De Bilt, Leeuwarden, Eelde, Rotterdam, Eindhoven, Maastricht for the Netherlands dataset.

As a preprocessing step, both datasets are normalized using the min-max normalization based on values coming from the corresponding training sets. The discussed datasets have been previously introduced in 3 and [7]. We train our model with two variants: with $\gamma$ set to 1 and with $\gamma$ learnt with other parameters. We evaluate our model together with the other models presented in [7]. The referenced models are trained and evaluated on the same datasets using the same data splits as used for our model. We report mean absolute error (MAE) for each model on the test set. The reported MAEs are obtained by taking the average over all output cities. The MAE is defined as follows: $M A E=\frac{\sum_{i=1}^{n}\left|y_{i}-\hat{y}_{i}\right|}{n}$ Here, $n$ denotes the number of samples in the test set. $y_{i}$ and $\hat{y}_{i}$ denote ground truth and predicted value respectively.

We train the models for predicting wind speed over 6, 12, 18 and 24 hours ahead for the Danish dataset and 2, 4, 6, 8 and 10 hours ahead for the Dutch dataset. The obtained MAE scores on the test set are tabulated in Table 1. The best scores are underlined for each prediction time for both datasets. For the Danish dataset, the scores of the baseline models are taken from the original paper [7]. For the Dutch dataset, the relevant scores of the baseline models are initially unavailable. We use the authors' publicly available code implementation to perform appropriate training and collect the corresponding MAE scores.

It can be observed that the proposed model WeatherGCNet with its both variants, when $\gamma$ is set to 1 and when $\gamma$ is learnt, outperforms all the other baseline models. This is due to the fact that our model learns and incorporates the spatial neighbor relations between the weather stations based on a graph rather than a tensorial form used in 3,7 , where neighborhood is determined 
ESANN 2021 proceedings, European Symposium on Artificial Neural Networks, Computational Intelligence and Machine Learning. Online event, 6-8 October 2021, i6doc.com publ., ISBN 978287587082-7.

Available from http://www.i6doc.com/en/.

Table 1: The average MAEs of evaluated models for wind speed prediction over the Danish and Dutch cities datasets.

\begin{tabular}{|c|c|c|c|c|c|c|c|c|c|}
\hline Model & \multicolumn{4}{|c|}{ Denmark } & \multicolumn{5}{|c|}{ Netherlands } \\
\hline & $6 \mathrm{~h}$ & $12 \mathrm{~h}$ & $18 \mathrm{~h}$ & $24 \mathrm{~h}$ & $2 \mathrm{~h}$ & $4 \mathrm{~h}$ & $6 \mathrm{~h}$ & $8 \mathrm{~h}$ & $10 \mathrm{~h}$ \\
\hline 2D & 1.304 & 1.746 & 1.930 & 2.004 & 8.18 & 10.08 & 12.03 & 13.15 & 14.51 \\
2D + Attention & 1.313 & 1.715 & 1.905 & 1.950 & 8.10 & 10.09 & 11.83 & 13.10 & 14.13 \\
2D + Upscaling & 1.307 & 1.723 & 1.858 & 1.985 & 8.24 & 10.22 & 11.83 & 13.74 & 14.80 \\
3D & 1.311 & 1.677 & 1.908 & 1.957 & 8.05 & 10.15 & 11.93 & 13.01 & 14.24 \\
Multidimensional & 1.302 & 1.706 & 1.873 & 1.925 & 8.10 & 10.03 & 11.46 & 12.79 & 13.81 \\
WeatherGCNet $(\gamma=1)$ & 1.279 & 1.638 & 1.777 & 1.869 & $\underline{7.96}$ & 9.97 & 11.16 & $\underline{12.30}$ & $\underline{13.33}$ \\
WeatherGCNet $($ learnt $\gamma)$ & $\underline{1.267}$ & $\underline{1.616}$ & $\underline{1.767}$ & $\underline{1.853}$ & 7.97 & $\underline{9.74}$ & $\underline{10.99}$ & 12.44 & 13.55 \\
\hline
\end{tabular}

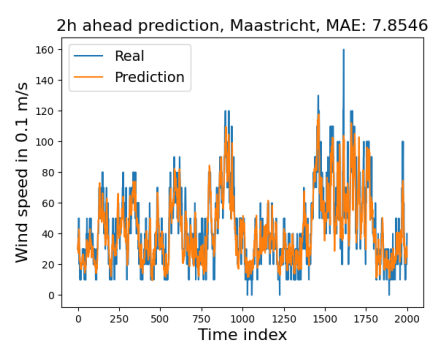

(a)

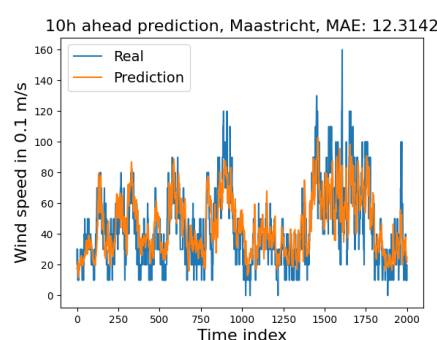

(b)

Fig. 3: WeatherGCNet $(\gamma=1)$ wind speed prediction plots for Maastricht (NL) for: (a) $2 \mathrm{~h}$ ahead, (b) $10 \mathrm{~h}$ ahead.

only by the order of the cities in the dataset. Fig 3(a) and 3(b) show plots of wind speed prediction obtained by the proposed model for the selected Dutch city Maastricht. It should be noted that a subset of test data is used for plotting whereas MAE scores included in the figures are reported for the whole test set. Further, we also visualize the learnt adjacency matrices of the proposed model for $2 \mathrm{~h}$ ahead wind speed prediction for the Dutch dataset. Fig. 4(a) shows the visualization of the adjacency matrix in the first spatio-temporal layer of the WeatherGCNet model, where $\gamma$ is set to 1. Fig. 4(b) shows analogous visualization for model where the $\gamma$ parameter is learnt. Given the possibility to decide via the additional parameter, the network prefers the self-loop connection to be relatively small (compared to the other entries), as visible in Fig. 4(b).

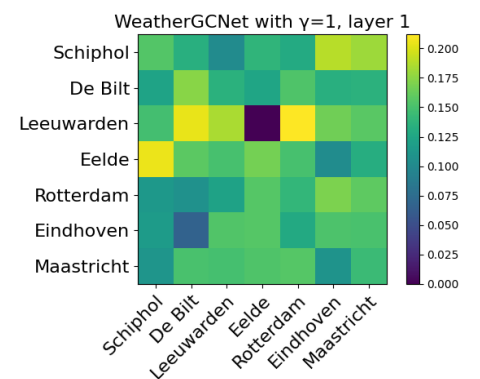

(a)

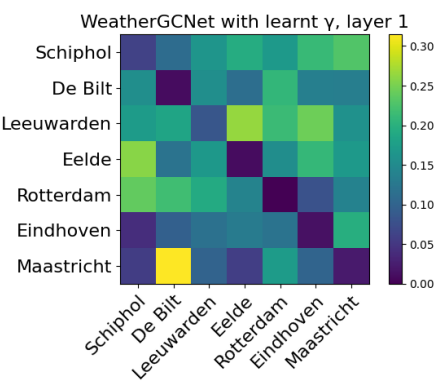

(b)

Fig. 4: Adjacency matrix (first layer) visualization for wind speed $2 \mathrm{~h}$ ahead prediction on Dutch dataset. (a): Model with $\gamma=1$; (b): model with learnt $\gamma$. 
ESANN 2021 proceedings, European Symposium on Artificial Neural Networks, Computational Intelligence and Machine Learning. Online event, 6-8 October 2021, i6doc.com publ., ISBN 978287587082-7.

Available from http://www.i6doc.com/en/.

\section{Conclusion}

In this paper, new model based on GCN architecture is proposed for wind speed prediction using historical weather data. Thanks to the applied spatialtemporal convolutional operations on the built graph, the network learns the underlying relations between weather stations through a learnable adjacency matrix of the graph. The self-loop connection of the adjacency matrix is enforced with and without learnable scalar parameter enabling the network to decide about the strength of the self connectivity in the graph. Comparing with previously introduced baselines, our proposed model provides more accurate predictions in all cases examined. The github implementation is available at https://github.com/tstanczyk95/WeatherGCNet

\section{References}

[1] Sebastian Scher. Toward data-driven weather and climate forecasting: Approximating a simple general circulation model with deep learning. Geophysical Research Letters, 45 , 112018.

[2] Jesús García Fernández, Ismail Alaoui Abdellaoui, and Siamak Mehrkanoon. Deep coastal sea elements forecasting using $\mathrm{u}$-net based models. arXiv preprint arXiv:2011.03303, 2020 .

[3] Siamak Mehrkanoon. Deep shared representation learning for weather elements forecasting. Knowledge-Based Systems, 179:120-128, 2019.

[4] Sepp Hochreiter and Jürgen Schmidhuber. Long short-term memory. Neural Computation, 9(8):1735-1780, 1997.

[5] Sima Siami Namini, Neda Tavakoli, and Akbar Siami Namin. A comparison of arima and lstm in forecasting time series. ICMLA, pages 1394-1401, 122018.

[6] Xingjian Shi, Zhourong Chen, Hao Wang, Dit-Yan Yeung, Wai-kin Wong, and Wangchun Woo. Convolutional lstm network: A machine learning approach for precipitation nowcasting. NIPS, pages 802-810, 2015.

[7] Kevin Trebing and Siamak Mehrkanoon. Wind speed prediction using multidimensional convolutional neural networks. arXiv preprint arXiv:2007.12567, 2020.

[8] Franco Scarselli, Marco Gori, Ah Chung Tsoi, Markus Hagenbuchner, and Gabriele Monfardini. The graph neural network model. IEEE Transactions on Neural Networks, 20(1):61-80, 2009.

[9] Si Zhang, Hanghang Tong, Jiejun Xu, and Ross Maciejewski. Graph convolutional networks: a comprehensive review. Computational Social Networks, 2019.

[10] T. Wilson, P. Tan, and L. Luo. A low rank weighted graph convolutional approach to weather prediction. In 2018 IEEE International Conference on Data Mining (ICDM), pages 627-636, 2018.

[11] M. Khodayar and J. Wang. Spatio-temporal graph deep neural network for short-term wind speed forecasting. IEEE Transactions on Sustainable Energy, 10(2):670-681, 2019.

[12] Sijie Yan, Yuanjun Xiong, and Dahua Lin. Spatial temporal graph convolutional networks for skeleton-based action recognition. AAAI, 2018

[13] Lei Shi, Yifan Zhang, Jian Cheng, and Hanqing Lu. Adaptive spectral graph convolutional networks for skeleton-based action recognition. CVPR, 2019.

[14] Thomas N. Kipf and Max Welling. Semi-supervised classification with graph convolutional networks. ICLR, abs/1609.02907, 2017. 